\title{
Linx
}

Revue des linguistes de l'université Paris X Nanterre

5 | 1994

La négation

\section{Ce n'est pas que, non que}

\section{Pierre Attal}

\section{OpenEdition}

\section{Journals}

Édition électronique

URL : http://journals.openedition.org/linx/1214

DOI : 10.4000/linx.1214

ISSN : 2118-9692

\section{Éditeur}

Presses universitaires de Paris Nanterre

\section{Édition imprimée}

Date de publication : 1 juin 1994

Pagination : 235-250

ISSN : 0246-8743

\section{Référence électronique}

Pierre Attal, «Ce n'est pas que, non que », Linx [En ligne], 5 | 1994, mis en ligne le 18 juillet 2012,

consulté le 19 avril 2019. URL : http://journals.openedition.org/linx/1214 ; DOI : 10.4000/linx.1214

Ce document a été généré automatiquement le 19 avril 2019

Département de Sciences du langage, Université Paris Ouest 


\title{
Ce n'est pas que, non que
}

\author{
Pierre Attal
}

\section{NOTE DE L'ÉDITEUR}

Tous les exemples sont réunis en annexe suivant la numérotation indiquée dans le texte.

1 Parmi les tournures négatives du français, il en est deux qui posent des problèmes délicats, car elles n'ont pas, si l'on peut dire, «l'air pur ». Il s'agit des locutions ce n'est pas que $p$, non (pas) que $p$. Elles ne sont pas très fréquentes et elles ont été peu étudiées ou de façon superficielle. On en parle dans les grammaires classiques (Grevisse, F. Brunot dans sa grande histoire du français dans le volume IV, 2e partie, consacrée au français du XVIIe s.), mais également dans des ouvrages modernes comme celui de D. Gaatone (1971) ou celui de C. Muller (1991). Le premier relève des spécimens écrits intéressants, Muller fait une analyse avec laquelle je serai en gros d'accord.Cependant, j'apporterai un soin particulier à situer ce n'est pas que par rapport à c'est que. L'étude de c'est que est encore plus difficile que celle de la négation; les formes marquées offrent plus de prise à l'analyse que les formes non marquées.

2 Je tâcherai de montrer que tant «c'est que $p$ » que «ce n'est pas que p» (ou «non que $\mathrm{p} »)$ sont des façons insistantes, emphatiques de dire « $\mathrm{p}$ » ou sa négation sous la forme classique (ne $V$ pas). La notion d'explication ou de cause à laquelle on les rattache d'ordinaire ne couvre pas tous les cas. Nous y verrons un effet dérivé.

Ce n'est pas que $p$, tout particulièrement, amplifie le caractère métalinguistique que j'attribue à tout opérateur de négation; il met l'accent sur la valeur «pédagogique » ou l'aspect ad hominem que Bergson déjà reconnaissait à la négation dans L'Evolution Créatrice. Mais d'autre part, les tournures partagent tous les effets reconnus généralement à ne pas. D'une certaine façon, elle laisse voir presque caricaturalement la dualité de niveaux d'une énoncé négatif : neg sur $p$, et rend encore plus sensible son caractère polyphonique.

4 Je ne parlerai que très brièvement du mode de $p$ après ce n'est pas que. D'abord, on ne trouve le subjonctif qu'après la négation et jamais après c'est que. Cela n'exige aucun 
éclaircissement. Quant à la répartition des formes à l'indicatif et au subjonctif après les locutions négatives, elle n'appelle pas non plus de commentaire particulier : le subjonctif est essentiellement écrit et littéraire, l'oral présente plutôt l'indicatif (dans la mesure où on peut distinguer les modes à l'oreille).

Un seul détail est à signaler : F. Brunot et Grevisse relèvent des exemples de ce n'est pas que avec l'indicatif au XVII ${ }^{e}$ s. et une valeur non négative, ou, si l'on préfère avec la valeur de ce n'est pas que ne pas. Les textes ne présentent aucune équivoque : (28) «Il leur fait verser à boire parle héraut (...) Ce n'est pas qu'il y admet encore d'autres valets ». « Si le titre ne vous plaît, changez-le ; ce n'est pas qu'il m'a paru le plus convenable ». «Ce n'est pas qu'il faut quelquefois pardonner à celui qui, avec un grand cortège, un habit riche et un magnifique équipage, s'en croit plus de naissance et plus d'esprit : il lit cela dans la contenance et dans les yeux de ceux qui lui parlent». Bien sûr, les auteurs ne risquent aucune explication, et je n'en ai aucune ${ }^{1}$.

6 Nous nous intéresserons de plus près au rapport entre « $\mathrm{Ce}$ n'est pas que je sois mécontent, mais cela m'irrite » et "Ce n'est pas qu'il soit là qui m'irrite ». Cette dernière tournure représente une mise en relief, une forme de focalisation. Elle exige un corrélatif (relatif ou que). Or ce n'est pas que $p$ se comporte comme une proposition autonome (comparer «Je ne suis pas à vrai dire mécontent, mais cela m'irrite »). Cependant, j'ai trouvé un exemple où les deux constructions se rapprochent sensiblement l'une de l'autre : dans Le Malade Imaginaire, II, V, Diafoirus dit en commençant le portrait de son fils (1) : «Ce n'est parce que je suis son père, mais je puis dire que j'ai sujet d'être content de lui ». Certes, d'une part, on ne peut pas remplacer ce n'est pas parce que par ce n'est pas que (avec ce dernier il contesterait sa paternité), d'autre part, on pourrait, après ce n'est pas parce que sous-entendre une suite comme «que je parle ainsi ». Il n'empêche que c'est une correction. Nous aurions peut-être tort de séparer totalement les deux constructions.

7 Par contre, nous ne pourrions pas classer tout à fait à part notre tournure et ce n'est pas pour inf: "Ce n'est pas pour me vanter, mais je suis fort». On peut paraphraser cet énoncé en «Ce n'est pas que je chercher à me vanter,... $»^{2}$. On peut aussi penser à ce n'est pas pour dire. Nous rapprocherons encore les emplois suivants de notre expression :

- Il est lâche.

- Ce n'est pas ça

ou

- Ce n'est pas qu'il soit lâche.

Ce dernier peut être complété par c'est que. Le problème ici est de savoir si le caractère sensiblement "polémique » de "Ce n'est pas ça » ou de "Ce n'est pas qu'il soit lâche » nous interdit de les mettre sur le même plan que (11) «Ce n'est pas que la France ait manqué à la fille d'Henri le Grand». Le rôle logique et argumentatif de ce dernier est évident. Pour ma part, comme je regarde avec méfiance la distinction polémique/ descriptif, ma première réaction est de répondre « Non ». Mais, d'autre part, on peut très bien soutenir que les tournures qui nous occupent sont des négations polémiques "déguisées »; car on peut recomposer chaque un énoncé interrogatif prêté à un tiers, auquel on répond négativement.

8 Enfin, nous rappellerons que la forme ce n'est pas sert dans certains cas de négations simples: «Ce n'est pas tout le monde qui dirait ça». Nous rapprocherons celle-ci d'exemples comme « Ce n'est que ...ne ...pas ».

9 Ce n'est pas que est-il l'envers de c'est que ? C'est le point le plus délicat de la question. La difficulté première à propos de ce point provient de ce que notre problème, surtout si l'on 
cherche à situer c'est que $p$ par rapport à ce n'est pas que $p$, ne peut en aucun cas être résolu par appel à de simples intuitions, probablement d'ailleurs parce que ces tournures sont relativement peu usitées, ou bien parce qu'il n'y a pas d'unité dans les différentes occurrences de c'est que, ce qui aboutirait à l'échec de ma tentative de rapprocher le positif du négatif. Qui plus est, et ce phénomène est lié à la remarque précédente, il est très difficile de manipuler les citations, par exemple, en substituant ce n'est pas que à c'est que, pour voir ce que cela donnerait. En bref, ce que je vais dire sur ce point reste fragile et n'est destiné qu'à attirer l'attention sur le problème. Ce qui s'impose d'abord, c'est le schéma ce n'est pas que p, (mais) c'est que. Exemple (2) : « Il la menaça de la battre si elle ne se taisait. Ce n'est pas que le bûcheron ne fût peut-être plus fâché que sa femme, mais c'est qu'elle lui rompait la tête». Cette configuration est claire et n'appelle aucun commentaire. Peut-on trouver l'ordre inverse? Quoique je n'aie pas rencontré d'exemples, on peut le concevoir sans peine :

- Alors, il est bien venu.

- C'est qu'il est intéressé, ce n'est pas qu'il nous aime particulièrement

Il n'est pas impossible de rapprocher ces exemples de ceux où c'est que, ce n'est pas que suivent si $p$ : «S'il est venu, c'est qu'il est intéressé, ce n'est pas qu'il nous aime ». Il faut tenir compte quand même du fait que si $p$ ici, avec la valeur «s'il est vrai que, si l'on doit admettre que ", rend obligatoire la suite sous la forme c'est que ou ce n'est pas que. En particulier, la suppression de c'est que entraîne une différence d'interprétation considérable.

L'impression prévaut en outre que c'est que introduit une explication. Mais ce n'est pas toujours le cas: «Si Pierre est là, c'est que Marie est partie » admet deux analyses: 1 ) « Pierre est là parce que Marie est partie », 2) « Pierre est là » et ce fait m'oblige à penser que Marie est partie. Les exemples que j'ai relevés mettent en évidence une grande variété de liens possibles avec ce qui précède c'est que $p$. Cette diversité est un signal clair : il n'est pas question de décrire c'est que $p$ en multipliant les effets de sens. Une telle entreprise va à l'encontre du but poursuivi. Elle montre au contraire qu'il faut rendre compte de l'unité d'emploi. J'étudierai donc successivement plusieurs exemples :

a) Agrippine, héroïne de la BD de C. Bretécher (Nouvel Observateur, 10-16 Sept 1992) se fait photographier par une amie dans des poses de mannequin; le résultat est piteux; elle gémit : «Ce n'est pas avec ça que je vais être sélectionnée pour le concours de Top Model de 16 ans Magazine. Et sa copine, peu charitable et de mauvaise foi, rétorque: "Je m'esscuse, mais c'est que tu as pris un sacré coup de vieux". On peut recomposer avant c'est que "Si c'est raté... », mais c'est un réaménagement destiné à retrouver le schéma classique.

b) Dans Le Malade Imaginaire, II, VIII, Argan veut faire parler sa petite fille Louison, cela donne ceci :

Argan : Ah, ah, petite masque, vous ne me dites pas que vous avez vu un homme dans la chambre de votre sœur?

Louison : Mon papa!

Argan : Voici qui vous apprendra à mentir (Il brandit des verges)

Louison (se jette à genoux) : Ah, mon papa, je vous demande pardon. C'est que ma sœur m'avait dit de ne pas vous le dire, et je m'en vais vous dire tout.

Là encore, on peut supposer que c'est "Si j'ai menti, c'est que... ». Mais c'est toujours une reconstitution. Les autres spécimens n'admettent plus cette analyse, si bien que cela fait beaucoup de possibilités.

c) Dans la même scène un peu plus loin, on a : 
Argan : Oh, çà, çà, je vous pardonne pour cette fois-ci, pourvu que vous me disiez bien tout.

Louison : Oh oui, mon papa.

Argan : Prenez-y bien garde au moins, car voici un petit doigt qui sait tout, qui me dira si vous mentez.

Louison : Mais mon papa, ne dites pas à ma sœur que je vous l'ai dit.

Argan : Non, non.

Louison: C'est, mon papa, qu'il est entré un homme dans la chambre de ma sœur comme j'y étais.

On est obligé cette fois de supposer que c'est que répond à «Ce que j'ai à vous dire ». Mais c'est suspect.

d) Dans Les Femmes Savantes, II, IX, Ariste demande à Chrysale où en sont les affaires d'Henriette et ce que sa femme Philaminte :

Ariste

A-t-elle consenti ? L'affaire est-elle faite?

Chrysale

Pas tout à fait encor.

Ariste

Refuse-t-elle?

Chrysale

Non.

Ariste

Est-ce qu'elle balance?

Chrysale

En aucune façon.

Ariste

Quoi donc?

Chrysale

C'est que pour gendre elle m'offre un autre homme.

Là, la tentation est forte de dire que c'est que répond à " Quoi donc? ". Mais, comme dans l'exemple précédent, on peut suggérer aussi que c'est que est paraphrasable par « Il y a que...».

e) Même pièce, III, V : Vadius a gaffé envers Trissotin :

Vadius

Je ne sais comment se fit l'affaire.

Trissotin

C'est qu'on fut malheureux de ne pouvoir vous plaire.

f) Le Barbier de Séville, III, V

Bartholo (à Figaro): Vous le prenez bien haut, Monsieur! Sachez que quand je dispute avec un fat, je ne lui cède jamais.

Figaro, lui tourne le dos : Nous différons en cela, Monsieur; moi je lui cède toujours. Bartholo (au Comte, déguisé) : Hein ? Qu'est-ce qu'il dit donc, bachelier?

Figaro: C'est que vous croyez avoir affaire à quelque barbier de village, et qui ne sait manier que le rasoir?

On peut paraphraser cet exemple en : la vérité serait donc que...?

g) Port des brumes (Simenon) :

«Vous étiez ici hier !» lui dit le commissaire (...) «Eh bien, s'impatienta Maigret. Eh bien quoi, grommela Louis, vous dites que j'étais ici ! C'est que j'y étais... »

h) Le même :

«Allez m'attendre à bord ! dit le commissaire à Lannec.

- C'est que...

- Quoi?

- Dans deux heures, nous n'aurons plus assez d'eau pour prendre le large... » 
11 D'une façon générale, il semble que l'on puisse paraphraser tous ces emplois de c'est que par « Il y a que.. ». «La réalité est que... ». Ajoutons à ces exemples les énoncés qu'on peut former quand on découvre quelque chose qu'on n'attendait pas: "Mais c'est qu'il pleut ! », « Mais c'est qu'il fait beau, qu'il ne fait pas beau ».

12 Les problèmes soulevés par cette expression sont aigus: d'abord, il ne semble pas que dans les textes étudiés on puisse substituer ce n'est pas que à c'est que. Or à l'inverse, la substitution de c'est que à ce n'est pas que dans les exemples que nous verrons plus loin est possible.

13 Cependant, ce n'est pas une raison pour poser ces tournures comme étrangères l'une à l'autre. Nous pouvons en effet considérer les emplois de c'est que comme caractéristiques d'« actes initiatifs » (voir sur cette notion Mœeschler 1985 : 93s, Callebaut $1991: 117 \mathrm{~s})$. On compren-drait pourquoi ce n'est pas que est difficile dans les contextes ci-dessus: il faut que l'on ait dit ou suggéré $p$, autrement dit, il faut un intermédiaire.

Plus délicat est le problème suivant: on trouve c'est que interrogatif (ex (f)) et, mieux encore, c'est que suivi d'une phrase négative (ex (h)) : parlerons-nous d'interrogation, de négation ou d'affirmation? Au contraire, avec ce n'est pas que les choses sont claires, il y a rejet pur et simple de $p$. Je pense que la réponse doit être qu'il y a bien dans c'est que $p$ ? une interrogation, et dans c'est que neg $p$ une négation. Si nous laissons l'interrogation de côté, car il n'y a pas en face de c'est que $p$ ? une tournure est-ce que $p$ ? que l'on puisse décrire comme le correspondant de ce n'est pas que $p$ en face de c'est que ne pas, puisque estce que $p$ ? est une façon normale d'interroger sur $p$, si donc nous laissons l'interrogation, nous décrirons la différence entre c'est que ne pas et ce n'est pas que $p$ en termes de fonctions discursives différentes et non en termes d'opposition : affirmation vs négation (voir sur ces questions de fonctions, Callebaut 1991, entre autres 156s). Nous constatons en effet que les tournures c'est que ne pas sont régulièrement remplaçables par non $p$, de même que ce n'est pas que $p$ (sauf cas de double négation). Par exemple : quelqu'un se trouve mal; on discute de ce qu'on l'on doit faire ; "Il ne faut pas faire les choses avec précipitation », dit l'un; à quoi un autre objecte : «C'est qu'il n'est pas bien", non pour justifier quelque chose, mais simplement pour rappeler la situation. Or on peut remplacer cet énoncé par «Mais il n'est pas bien! $»^{3}$.

Par conséquent, avec c'est que ne pas nous ne parlerons pas d'affirmation. Le problème alors se déplace : c'est que ne peut plus être décrit comme introducteur d'affirmation, alors que nous décrirons bien ce n'est pas que comme une forme de négation. Nous en revenons à une sorte de dissymétrie entre la forme positive et la forme négative. Le caractère plus flou, moins tranché du positif ne nous étonnera pas. Il est naturel avec les formes non marquées. Ou bien encore nous poserons, à un niveau différent, un couple antithétique c'est que/ce n'est pas que, en rapport avec l'opposition affirmation/négation, mais différent cependant $d u$ couple $« p » / «$ non $p »$. Nous nous pencherons sur cette question plus loin.

Revenons à notre tournure négative; un problème mineur se pose: les distributions respectives de ce n'est pas que et de non (pas) que. Elles ne sont pas équivalentes: seul ce n'est pas que semble pouvoir ouvrir un discours, non que serait toujours second. Muller suggère que c'est lié à une propriété d'anaphore de non (1991: 189). Le terme n'est pas clair ; est-ce à dire que non est toujours une réponse, renvoie systématiquement à quelque chose d'antérieur? En supposant qu'on ne tienne compte que des non phrastiques, peuton dire que le cri «Non!» devant un spectacle qu'on n'admet pas est une anaphore? Je 
préférerais une solution syntaxique. Le fait que non que ne puisse intervenir en tête de discours tiendrait à ce qu'avant que qui doit introduire une phrase on doive avoir une structure phrastique claire. C'est le cas de ce n'est pas que. Au milieu d'un texte, non que peut être rattache à la structure phrastique antérieure, le point ou le point-virgule ayant une valeur de rupture à l'écrit et non à l'oral. En tout cas, cette interdiction n'a pas toujours été respectée ; voici un texte de Marot (16) où non que introduit l'épitre ; on peut toujours penser que c 'est une trace dialectale, Marot ayant parlé le patois du Quercy avant le français :

Non que par moy soit arrogance prinse,

Non que ce soit par curieuse emprinse

D'escrire au Roy ; pour tout cela ma plume

D'ardent désir de voler ne s'allume.

Mon triste deuil seulement l'a contraincte

De faire à vous, et non de vous complaincte.

(Epître au Roy pour succéder en l'estat de son père)

On a spontanément tendance à voir dans la tournure que nous examinons 1) une expression directement liée à la cause, 2) une partie d'une structure plutôt complexe et relativement stable. Nous nous pencherons sur ces deux caractéristiques pour les aménager sensiblement.

L'idée que cette expression relève de la cause est appuyée par une certain nombre de faits : on trouve ce n'est pas que $p$, mais parce que $q$ : (3) « Je continuai, non qu'il restât rien à ajouter, mais parce que je ne pouvais supporter son silence», (4) «Je pris plaisir à les observer, non qu'ils eussent rien d'extraordinaire, mais parce que je leur trouvai cet air brave et joyeux qui est naturel à la jeunesse ". Si nous regardons la citation de Marot cidessus, on remarque que les négations sont résumées par la négation de pour tout cela qui a une valeur causale. La tournure française a un ancêtre latin : non quo, non quod... sed (quia) où quod et quia sont identifiés comme des conjonctions de cause. Enfin, que en ancien français et en moyen français avait souvent une valeur de cause :

Et nuit et jour je chante

Son trépas dans ces bois, nommant la mort méchante,

Qu'elle ne m'a tuée avecques ma fidelle

Ronsard

Tout cela explique que Grevisse étudie notre locution dans les propositions de cause, comme Brunot dans sa grammaire du XVII ${ }^{\mathrm{e}}$. A présent, il faut aussi tenir compte des intuitions : dans un bon nombre de nos spécimens le rapport entre non que et ce qui précède peut être dit un rapport de cause (rejetée) à effet :

(5) « Alors je me suis dit que j'irais me coucher : non pas que j'avais sommeil. Il n'était que huit heures »

(6) « Et moi, ça m'agaçait. Pas que j'aimais, pas que j'enviais Migraine, et pourtant... »

(7) « Alors on avait soupiré quand les Bourbons étaient revenus, pas qu'on fût si royaliste que ça disait, mais... »

Nous en citons d'autres dans le relevé des exemples fait à la fin de l'article.

Cependant, je critiquerai toute caractérisation première de ce n'est pas que $p$ en termes d'expression de la cause : d'abord on trouve peu souvent parce que aussi bien en tête qu'en correctif. Si on en revient à l'exemple de Diafoirus que j'ai cité : « Ce n'est pas parce que je suis son père, mais je puis dire que...", si on n'analyse pas cet énoncé comme une clivée avec une partie sous-entendue, on s'aperçoit qu'on ne peut quand même pas substituer à parce que $p$, que $p$ tout court. Mais cela reste forcément sujet à caution. Voici un exemple 
plus probant: (26): «S'il trouve des mots aussi justes pour réduire à coups de sourires son cancer à une banalité du destin (...), ce n'est pas parce qu'il puise dans le terreau de son savoir-faire en cherchant une fois de plus à désarmer ceux qui lui reprochent le confort de sa solitude souveraine ", où je ne crois pas qu'on puisse remplacer que par parce que. Il y a autre chose : tant en ancien français qu'en français moderne que ne peut pas être tenue pour une conjonction de cause ; que n'a aucune couleur, il est neutre. De plus, un fait m'a frappé : Foulet dans sa Syntaxe de l'ancien français dit à propos de que appelé causal que la conjonction est surtout littéraire et que les écrits les plus proches de l'oral emploient de préférence car, qui n'est pas à proprement parler d'une conjonction de cause, mais introduit une justification.

Il y a surtout bien sûr le fait que beaucoup d'exemples n'ont rien à voir avec la cause : (10) "Entre mes voyages, séparés seulement de quelques années, il y avait de moins une monarchie de quatorze siècles. Ce n'est pas que j'en veuille le moins du monde à ces révolutions politiques... », (12) « Racine se vante d'avoir, même en une comédie, réjoui le parterre "sans qu'il lui ait coûté une seule de ces sales équivoques et de ces malhonnêtes plaisanteries qui coûtent maintenant si peu à la plupart de nos écrivains" (...) Ce n'est pas que Racine, dans son œuvre, ait partout et toujours cherché le mot noble...»; (15) »E. Balladur. Assurément le plus européens des dirigeants du RPR. Non pas qu'E. Balladur puisse être considéré comme un Euromaniaque »; voir encore les ex. (17), (20), (21), (22), (23), (24), (25). D'ailleurs, de manière significative, Grevisse se contredit dans l'édition de 1969 dont je me sers (p. 1086). Il parle de l'indicatif et dit : »A l'époque moderne, ce n'est pas que, au sens de "On ne doit pas dire, à cause de cela, que..." se rencontre avec l'indicatif ». Autrement dit, il inverse le rapport; la négation devient la conséquence, et en plus le rapport ne se situe pas du tout au même niveau. Il est frappant que la paraphrase utilisée par Grevisse soit formellement proche de la locution employée par Séchehaye: (27) »Ce n'est pas à dire qu'une langue ne porte pas les caractères correspondant à ceux de la collectivité qui l'a créée, mais... ».

21 Pour rendre compte d'au moins une bonne part de ces emplois, il parait plus juste de dire que l'énonciateur cherche à empêcher, en prenant les devants, que le destinataire tire une conclusion d'un énoncé qu'il a émis ou simplement que ce dernier propose telle description d'une situation constatée. On peut souvent remplacer ce n'est pas que par « $\mathrm{Ne}$ croyez surtout pas que » ou «Ne concluez pas que ». Nous verrons que l'on peut élargir encore cette caractérisation et réduire considérablement l'écart entre ce n'est pas que $p$ et ne pas. Nous sommes donc plutôt dans le domaine de l'argumentation, comme l'a vu C. Muller, du moins si nous laissons de côté certains exemples dont nous parlerons plus tard. Une conclusion peut être dans des rapports divers avec sa prémisse, y compris un rapport de cause à effet. Il n'y a donc rien d'étonnant à ce que nous ayons souvent l'impression que ce qui suit ce n'est pas que est une cause rejetée d'un fait cité auparavant.

Nous nous intéresserons maintenant au second aspect qui permet de voir dans la tournure un élément d'une structure relativement spécialisée, voire stable: le fait que l'on attende régulièrement une contrepartie correctrice. Or là encore la situation n'est pas simple ; il y a plusieurs combinaisons possibles : d'abord, nous avons le remplacement de l'énoncé écarté par un autre, comme dans (2) «Il la menaça de la battre si elle ne se taisait. Ce n'est pas que le bûcheron ne fût peut-être plus fâché que sa femme, mais c'est que elle lui rompait la tête »; voir encore (3) et (4). (7) « Alors on avait soupiré quand les Bourbons étaient revenus, pas qu'on fût si royaliste que ça se disait, mais l'ordre, la monarchie, si cela avait pu apporter avec la paix, une vie normale, enfin du travail » est 
moins clair, mais semble construit de la même façon : « on n'était pas si royaliste que ça ", mais (on disait) «l'ordre, la monarchie, si cela pouvait apporter...", autrement dit, on oppose les deux explications du soulagement. C'est sans doute le cas de (13) «Depuis, le vétéran de la diplomatie mondiale éprouvait quelque peine à redessiner une politique extérieure pour l'Allemagne unie (...) Non que les dogmes fondateurs ne demeurent (...) Mais les trois piliers traditionnels de la diplomatie allemande ont été ébranlés ", où sont opposés «les dogmes fondateurs ne demeurent (pas)» et "les trois piliers ont été ébranlés ». C'est le schéma de ce début d'une vieille chanson d'Y. Montand (20) :

«C'est pas qu'elle est belle, belle,

Elle a pas d'bijoux, ni de fines dentelles,

Mais elle a en elle

Quelque chose qui plaît "

Dans ces exemples, de manière sûre en tout cas dans les trois premiers, le mais est plutôt un mais "sino" (mais SN selon la classification d'Anscombre et Ducrot), ce que m'a confirmé pour (2) et (4) Hermandez-Paricio. Autrement dit, chaque fois que nous avons bien substitution d'une conclusion-cause à une autre et qu'elles sont mises sur le même plan, nous avons mais SN. Cependant, non informateur précise qu'il suffit que le parallélisme entre les deux énoncés soit moins marqué, et donc que le second énoncé soit présenté comme un argument meilleur, sans être affirmé de manière explicite comme une cause, pour que la conjonction soit pero: exemple, «Ce n'est pas que (No es que) le bûcheron ne fût pas plus fâché que sa femme, mais (pero) elle lui rompait la tête », sans c'est que après mais. Dans ce cas, ce n'est pas ou no es que n'est plus rattaché à l'idée de cause de manière claire, mais vaut je ne dis pas que (no digo que). C'est probablement ainsi qu'on doit interpréter (7), (13) et (20), contrairement à ce que j'ai supposé.

Il est certain au moins que c'est le schéma des autres énoncés contenant mais $q$ ou pourtant q. Exemple (6) : «Et moi, ça m'agaçait. Pas que je l'aimais, pas que j'enviais Migraine, et pourtant quand il me parlait d'elle, j'en éprouvais du dépit ». Le lecteur/ destinataire pourrait conclure de «Et moi, ça m'agaçait: "Donc il aime Migraine", je rejette cette conclusion; il n'y a cependant aucune contradiction entre non $q$ («Pas que j'aimais Migraine ») et $p$ (" Ça m'agaçait»). Ce qui suit et pourtant redit d'une autre façon ce que disait $p$. Autre exemple (11) : «Elle fut contrainte de paraître au monde et d'étaler, pour ainsi dire, à la France même et au Louvre, où elle était née avec tant de gloire, toute l'étendue de sa misère (...) Ce n'est pas que la France ait manqué à la fille d'Henri le Grand ; Anne la magnanime, la pieuse (...) la reçut d'une manière convenable à la majesté des deux reines. Mais les affaires du Roi ne permettant pas que cette sage régente pût proportionner le remède au mal, jugez de l'état de ces deux princesses (Henriette et sa fille) »: Henriette était malheureuse, conclusion possible : la France a manqué à la fille d'Henri le Grand; je rejette cette conclusion et mon refus s'appuie sur le fait qu'Anne l'a bien reçue. Quant à mais, il introduit non pas l'invitation ("jugez de l'état...»), mais la participiale: "Les affaires du roi ne permettant pas que... ». Il est donc équivoque, car cette dernière proposition peut être dite aussi bien substituée à $p$ (« la France a manqué à Henriette ») qu'opposée argumentativement à «Anne l'a bien reçue ». Nous pourrions faire une analyse proche de celle de (6) pour (12) et (9) : « Il se contentait (...) d'inspecter de loin si tout allait bien (...) Ce n'est pas qu'il n'eût su, bien qu'il cachât ses débuts comme plongeur, mettre la main à la pâte comme un autre. Il fallut pourtant une circonstance exceptionnelle pour qu'un jour il découpât les dindonneaux.». Dans ces exemples, nous avons le schéma : 


\begin{tabular}{|l|l|}
\hline plan de l'explicite : & plan de l'implicite : \\
\hline$p$ & $p \rightarrow q$ \\
\hline non $q$ & non $q \rightarrow r$ \\
\hline \hline & $(r$ contredit $p)$ \\
\hline pourtant, mais $r$ ' & \\
\hline$\left(r^{\prime}\right.$ vaut $\left.p\right)$ & \\
\hline
\end{tabular}

Dans tous ces cas, nous avons un mais PA (pero-aber).

Schéma de même type, mais plus simple, toujours avec mais PA (pero/aber) (17) :

"C'est pas qu'il est beau,

Qu'il est riche, ni costaud,

Mais je l'aime»

où " Mais je l'aime " ne corrige en aucun cas "C'est pas qu'il est beau ", ce qui nous rapproche considérablement de «Il n'est pas beau, mais (pourtant) je l'aime ». Ajoutons ces exemples dont les deux premiers sont imaginaires, mais parfaitement familiers : (18) «C'est pas que ça m'arrange, mais je peux me débrouiller », (19) «Bon, c'est pas que je m'ennuie, mais il faut que je m'en aille », (21) "Les leaders des territoires occupés pourront désormais se réclamer ouvertement de la centrale dirigée par M. Yasser Arafat. Ce n'est pas d'ailleurs qu'ils se gênaient, mais enfin, théoriquement, M. Fayçal Husseini et Mme Hanan Achraoui risquaient la prison ». Quand on fait bien attention, on se rend compte que tous ces schémas se retrouvent avec une négation sous la forme classique ne pas. Par conséquent, il n'y a rien là qui caractérise la tournure de façon vraiment exceptionnelle.

Enfin, nous avons ce n'est pas que p sans suite (8) : «Aussi, je refusai de souper. Ce n'est pas que je ne me plusse chez la Princesse de Guermantes. Ainsi les hommes peuvent avoir plusieurs sortes de plaisirs. Le véritable est celui pour lequel ils quittent l'autre ", qui cependant est plus subtil, car, s'il n'y a pas une contrepartie à « Ce n'est pas que je ne me plusse », sous la forme "C'est que», ou "Mais...», cette contrepartie évoquée par l'expression "plusieurs sortes de plaisirs» et «le véritable est celui...» a bien été exprimée avant sous la forme « Albertine devait venir chez moi aussitôt après le théâtre » sur quoi l'auteur enchaîne par notre texte. L'essentiel pour nous ici, c'est que le jeu d'oppositions entre les énoncés n'entre pas dans la configuration ce n'est pas que $p$, mais (que) q. (10) «Entre mes voyages, séparés seulement de quelques années, il y avait de moins une monarchie de quatorze siècles. Ce n'est pas que j'en veuille le moins du monde à ces révolutions politiques ; en me rendant ma liberté, elles m'ont rendu à ma propre nature " ne présente rien après la négation. Même chose pour (15), et (22) «L'acceptabilité est à première vue un concept non théorique; elle n'est rien d'autre qu'une valeur. Cela n'empêche pas le linguiste d'avoir à construire une représentation adéquate du type de jugement correspondant, même s'il le fait de manière discutable (...) Ce n'est pas pour autant que la grammaire devient T-1 normative... ». Avec (14) « Le quai d'Orsay accuse le coup. Non que le tort causé à l'image de la France à l'étranger soit ressenti comme irréparable: on déplore tout juste quelques sarcasmes des amis 
britanniques (...) Non que la controverse soit engagée sur l'attitude de la France à l'égard des Palestiniens : l'histoire dira peut-être un jour ce que la venue à Paris du leader du FPLP avait à voir avec une politique qui... ", il y a deux fois une espèce de correction, mais elle n'est pas argumentativement opposée. C'est donc un nouveau cas.

J'ai donc essayé de mettre en évidence que les relations entre $p$ et ce n'est pas que $q$ ne se ramènent pas seulement à celle de cause à effet et de souligner la diversité des schémas où peut entrer la locution ce n'est pas que. J'ai voulu montrer que l'on ne pouvait pas y voir un élément d'une structure bien typée, à classer à part. Mes arguments ont été surtout négatifs. A présent, je vais avancer des arguments en faveur de l'idée que, si ce n'est pas que est une forme très marquée, insistante, emphatique de la négation, ce qui limite ses contextes possibles, elle réalise fondamentalement les valeurs classiques de ne pas.

1) Un argument que je ne pense pas avoir trouvé ailleurs : ce n'est pas que est une tournure commode pour introduire une double négation. Comme il est pratiquement impossible d'avoir deux fois ne pas ou pas dans la même phrase, on a recours à des périphrases : c'est le cas de (2) (8), (9), (13) « Depuis, le vétéran de la diplomatie mondiale éprouvait quelque peine à redessiner une politique extérieure pour l'Allemagne unie (...) Non que les dogmes fondateurs ne demeurent (...) Mais les trois piliers traditionnels de la diplomatie allemande ont été ébranlés. », (25) «Quant à la Nomanklatura, ce n'est pas qu'elle n'aime pas Gorbatchev, c'est qu'elle le hait », (27). Dans l'édition de 1969 de Grevisse, il y a encore d'autre exemples. Autrement dit, déjà, on peut soutenir que le recours à ce n'est pas que est justifiable par des impératifs syntaxiques.

2) Comme je l'ai dit il y a un instant, le rôle argumentatif de ce n'est pas que, empêcher une conclusion, est celui de ne pas. Ce n'est pas une nouveauté. Si nous prenons (10), un effet similaire peut être obtenu par «Je n'en veux pas le moins du monde à ces révolutions ». Il manque pourtant quelque chose ; l'articulation serait plus claire avec pour autant. Or si ici il apparaît que ce n'est pas que est bien remplacé par ne pas pour autant, dans (22) pour autant est déjà présent; autrement dit, nous nous rapprochons d'autant plus dans cet exemple de ne pas. J'ai depuis remarqué qu'à l'oral on emploie assez fréquemment ce n'est pas pour autant que. Donc, c'est une simple variante de ne pas pour autant. On peut dire la même chose de (21), où d'ailleurs rend plus aisé le remplacement de la locution négative par le double morphème classique (" d'ailleurs, ils ne se gênaient pas »). D'ailleurs et pour autant jouent un rôle argumentatif qui a été bien mis en évidence par Ducrot et d'autres.

Le parallélisme entre les deux formes de négation est bien souligné dans (20) par l'enchaînement : « C'est pas qu'elle est belle, elle n'a pas de bijoux ».

Enfin, ce n'est peut-être pas un hasard si ces dernières formes où le parallélisme entre ce n'est pas et ne pas est le plus sensible sont précisément celles où l'on trouve le plus naturellement l'indicatif.

31 3) Très proche de ne pas, cet emploi de ce n'est pas que, dans un dialogue, pour compléter un « Non»:

- Je voudrais bien aussi, Madame, répondis-je, que cela ne fût pas ; et je sens, à la peur étrange que vous en avez, combien vous me rendriez malheureux.

- Non, reprit-elle, ce n'est pas que j'en aie peur; craindre de vous voir amoureux serait avouer à demi que vous pourriez me rendre sensible : l'amant que l'on redoute le plus est toujours celui que l'on est le plus près d'aimer ; et je serais bien fâchée que me crussiez si craintive avec vous. (Crébillon, Les Egarements du cœur et de l'esprit). 
4) Dernier argument, à mes yeux très significatif : même si c'est un phénomène rare, on trouve ce n'est pas que avec une valeur que j'appelle non contre-argumentative, que d'autres qualifient de "métalinguistique » : c'est le fait que la négation est suivie d'un énoncé qui corrige la proposition dans un sens renforçateur, et non minorant ; j'ai relevé deux exemples oraux (23):

- C'est une question de biorythme.

- Tu crois au biorythme?

- Ce n'est pas que j'y croie (crois). Cela existe. et surtout (25).

Un autre exemple, plus subtil, nous est offert par un dialogue du film «La femme du boulanger »; il est nettement "métalinguistique »: Raimu-le boulanger n'a pas été réveillé par sa femme, et sa fournée a brûlé ; au client qui le tire de son sommeil en lui disant "Je crois que vous êtes endormi ", il réplique: "Ce n'est pas que je me suis endormi; je ne me suis pas réveillé». C'est un spécimen typique de déplacement d'argumentation; il ne s'agit pas de nier la réalité, mais de changer le type de reproche; en gros, le boulanger veut dire: «Il ne faut pas me faire des reproches sous la forme "Vous vous êtes endormi", car il est normal que je me sois endormi; mais il faut me reprocher de ne m'être pas réveillé ».

A ce point, le problème est: dans les derniers $\$$, a-t-on affaire au même ce n'est pas que qu'en (2), (3), etc. ? Après tout, j'ai bien écarté la tournure ce n'est pas Pierre qui... Donc, en soi, rien n'interdit de parler d'un second ce n'est pas que. Mais a) l'identité dans notre domaine est fonction du point de vue, c'est-à-dire de la théorie. Dans ce cas, le problème se ramène à : ma théorie, qui refuse de séparer radicalement les deux types de négations (appelées, à tort, linguistique et métalinguistique), est-elle valable ? Mais nous quittons la stricte question de la description de ce n'est pas que ; b) si on dit qu'il y a deux ce n'est pas que, on peut être amené à en distinguer encore plus, ce qui est théoriquement fâcheux. Je constate en effet que «Ce n'est pas qu'ils se gênaient d'ailleurs » ou le suivant sont assez éloignés intuitivement de (2) ou d'autres, que «C'est pas qu'elle est belle » ou «C'est pas qu'il est beau » ne semblent pas tout à fait assimilables à, disons, (12) ou (13). A cette division qui est entièrement tributaire des intuitions, je préfère toujours la voie de l'unité, au moins à une étape précoce de la théorie, quitte à ménager des bifurcations plus bas.

Par rapport à notre question, les bifurcations prendraient plusieurs formes : si avec ce n'est pas que nous avons une tournure négative fondamentalement équivalente de ne pas, elle présente l'originalité d'être clairement métalinguistique, elle détaille les deux niveaux que je pense être ceux de toute négation: un énoncé virtuel et le rejet de cet énoncé. La forme syntaxique a l'avantage d'isoler les deux parties; dans mon jargon, la première, l'énoncé possible prévu par l'énonciateur, est une suite locutionnaire; j'entends par là quelque chose de différent de ce que dit Austin; le locutionnaire est la forme syntaxique d'énonciations possibles, non prises en charge par l'énonciateur ou dont le contenu ne correspond pas aux intentions de celui qui parle, soit parce qu'il s'agit d'énoncés réels ou virtuels d'un tiers, soit parce qu'on pense que ce qu'apporterait l'énonciation directe est sans intérêt ou superflu; dans « C'est pas qu'il est beau » il est beau est une suite locutionnaire; quelqu'un pourrait dire à propos de « il » qu'il est beau, ou s'attendre à ce que l'énonciateur (la chanteuse) le dise. La seconde partie est le niveau de l'illocutionnaire, c'est-à-dire ce qui correspond aux intentions de celui qui parle, ce 
qu'il prend en charge en tant qu'énonciateur. Ici, il s'incarne dans les formes négatives. Nous avons donc un exemple caractéristique de ce qu'on a appelé la polyphonie.

D'autre part, la séparation syntaxique des deux niveaux a l'avantage de conserver au locutionnaire sa forme originelle. En disant "C'est pas qu'elle est belle», on évite les problèmes créés par l'introduction de la négation-morphème dans la proposition elle est belle. Celui qui parle ainsi ne risque pas de faire comprendre "Elle est laide». Remarquons aussi que lorsque la confusion n'est plus possible, on récupère la tournure normale : «Elle n'a pas de bijoux ». Même chose pour " Pas que j'aimais Migraine », qui se distingue clairement du composé négatif ne-pas-aimer = "détester ». Nous avons donc avec cette construction la valeur essentielle de la négation qui n'est pas d'indiquer le contraire, mais de rejeter sans rien indiquer.

Comme je l'ai dit, cette forme marquée de négation, même si elle est moins marquée que il n'est pas vrai que, a une tendance naturelle à une sorte de spécialisation; on a pu voir cependant qu'elle ne s'enferme pas dans un rôle trop rigide. Il n'est pas impossible que ce n'est pas que comme c'est que, non causal, soient essentiellement employé dans des conversations ou des quasi conversations. Le caractère direct de la formule est sensible et se prête aisément à ces emplois. Dernière remarque : il est significatif que cette forme typiquement métalinguistique s'accompagne si rarement d'une valeur dite «métalinguistique " («Il n'est pas riche, c'est un Crésus»). Pour moi, au moins, cela souligne la faiblesse de la dernière catégorie.

Nous allons revenir au problème que nous avons évoqué au début des rapports entre c'est que ne pas et ce n'est pas que. Si nous soutenons que la première tournure est comme la seconde une forme de négation, comme elles ne s'utilisent pas indifféremment, nous devons rendre compte de la différence, et, d'autre part, comment articulerons-nous le fait que la première se présente d'abord comme un énoncé affirmatif avec son caractère de négation? On ne peut répondre qu'aux deux questions en même temps, car le caractère particulier de c'est que ne pas est précisément dû au fait qu'elle combine une forme syntaxique positive (c'est que) et un énoncé négatif.

L'examen des différents exemples de c'est que est instructif; nous ne sommes pas arrivés à une caractérisation nette; nous nous sommes contentés d'y voir une forme d'insistance, une façon de souligner un fait quelconque (à titre de cause, de justification, d'objection, d'aveu, etc.). Dans tous les cas, il s'agit d'imposer le fait. Mais, dira-t-on, il existe donc des faits négatifs, puisqu'on relève " C'est que dans deux heures il n'y aura pas assez d'eau »? Disons plutôt, et cela n'interdit nullement une description unitaire de la négation, qu'un fait, c'est-à-dire l'« image » intellectuelle d'un événement ou d'une réalité, s'exprime aussi bien sous la forme d'un énoncé négatif que sous la forme d'un énoncé positif. La différence entre "Il n'y a pas assez d'eau », réponse " polémique » à " Tu as assez d'eau » et l'énoncé spontané de même forme ou sous la forme "C'est qu'il n'y a pas assez d'eau » doit être représentée à un autre niveau que la caractérisation la plus générale de la négation. Ce sont deux entreprises différentes. Un modèle génératif de la négation doit ignorer au départ l'opposition: polémique/non polémique ("descriptif»). Mais autre chose est de rendre compte de l'utilisation dans le dialogue de la négation, comme le fait Callebaut (1991).

Par conséquent, l'opposition entre c'est que et ce n'est pas que ne serait pas à décrire, contrairement à ce que j'ai pu penser un moment, comme le correspondant de l'opposition la plus générale " $\mathrm{p}$ » et "non $\mathrm{p}$ », mais comme l'opposition entre deux modes discursifs : dans un cas, il s'agit de faire en sorte que l'interlocuteur tienne compte 
d'un fait (quelle que soit sa présentation, positive ou négative); dans l'autre, nous trouvons la valeur que nous plaçons en premier dans la description de la négation: empêcher quelqu'un de dire quelque chose, indifféremment encore, un énoncé affirmatif ou négatif, puisque ce n'est pas que permet une double négation. Nous retrouvons donc à un niveau différent de la description l'opposition, que nous refusons d'introduire précocement, entre négation "descriptive " (qui ne se distingue plus très nettement d'affirmations) et négation "polémique ». Ce n'est pas que serait une forme spécialisée de ce dernier mode.

\section{BIBLIOGRAPHIE}

Babby, L., 1980, Existential Sentences and Negation in Russian, Ann Arbor, Karoma Publishers.

Dahl, Ö., 1973, »On the Semantics of Quantified Noun Phrases and Related Problems», Göteborg, Département de linguistique générale, Université de Göteborg.

Heldner, C., 1989, «Le sujet grammatical dans la construction impersonnelle», Studia Neophilologica, 61, pp. 89-115.

Heldner, C., 1992, «Sur la quantification négative», Langue française, 1992, 94, pp. 80-92.

Kesik, M., 1986, «Description de la phrase impersonnelle entre la syntaxe, la sémantique et la rhétorique» in Actes du XVIIe congrès international de linguistique et philologie romanes, pp. 251-263, Publications de l'Université de Provence.

Kleiber, G., 1981, Problèmes de référence : Descriptions définies et noms propres. Recherches linguistiques. Etudes publiées par le Centre d'Analyse Syntaxique de l'Université de Metz. Paris, Klincksieck.

Mel'cuk, I.A. \& A.K. Zolkovskij, 1970, «Toward a Functioning 'Meaning-Text' Model of Language», Linguistics, 57, pp. 10-47.

Picabia, L., 1987, «Quand y a-t-il générique ? » in Rencontre(s) avec la généricité. Recherches linguistiques XII, G. Kleiber (éd.), pp. 235-258, Paris, Klincksieck.

Reboul, A., 1988/1989, «Pragmatique de l'anaphore pronominale», in SIGMA, 12-13, pp. 197-231, Publications de l'Université de Provence.

Reboul, A., 1989, «Résolution automatique de l'anaphore pronominale» in Modèles de discours, pp. 173-192, Berne-Francfort-Paris, Peter Lang.

Sperber, D., \& Wilson, D., 1986, Relevance. Communication and Cognition, Oxford, Basil

\section{ANNEXES}




\section{Liste des exemples auxquels il est fait référence par un numéro}

(1) Ce n'est pas parce que je suis son père, mais je puis dire que j'ai sujet d'être content de lui.

Molière (Le Malade Imaginaire, II, V)

(2) Il la menaça de la battre si elle ne se taisait. Ce n'est pas que le bûcheron ne fût peutêtre plus fâché que sa femme, mais c'est qu'elle lui rompait la tête.

Perrault (Le Petit Poucet)

(3) Je continuai, non qu'il restât rien à ajouter, mais parce que je ne pouvais supporter son silence.

Gide (La Symphonie Pastorale)

(4) Je pris plaisir à les observer, non qu'ils eussent rien d'extraordinaire, mais parce que je leur trouvai cet air brave et joyeux qui est naturel à la jeunesse.

A. France (Le Crime de S. Bonnard)

(5) Alors je me suis dit que j'irais me coucher ; non pas que j'avais sommeil. Il n'était que huit heures.

L. de Vilmorin (Migraine)

(6) Et moi, ça m'agaçait. Pas que je l'aimais, pas que j'enviais Migraine, et pourtant quand il me parlait d'elle, j'en éprouvais du dépit.

Ibid

(7) Alors on avait soupiré quand les Bourbons étaient revenus, pas qu'on fût si royaliste que ça se disait, mais l'ordre, la monarchie, si cela pouvait apporter avec la paix, une vie normale, le travail.

L. Aragon (La Semaine Saine)

(8) Aussi, je refusai de souper. Ce n'est pas que je ne me plusse chez la Princesse de Guermantes. Ainsi les hommes peuvent avoir plusieurs sortes de plaisirs. Le véritable est celui pour lequel ils quittent l'autre.

M. Proust (Sodome et Gomorrhe)

(9) Il se contentait (...) d'inspecter de loin si tout allait bien (...). Ce n'est pas qu'il n'eût su, bien qu'il cachât ses débuts comme plongeur, mettre la main à la pâte comme un autre. Il fallut pourtant une circonstance exceptionnelle pour qu'un jour il découpât les dindonneaux.

Ibid.

(10) Entre mes voyages, séparés seulement de quelques années, il y avait de moins une monarchie de quatorze siècles. Ce n'est pas que j'en veuille le moins du monde à ces révolutions politiques; en me rendant à la liberté, elles m'ont rendu à ma propre nature. Chateaubriand (Mémoires d'Outre-Tombe)

(11) Elle fut contrainte de paraître au monde et d'étaler, pour ainsi dire, à la France même et au Louvre, où elle était née avec tant de gloire, toute l'étendue de sa misère (...) Ce n'est pas que la France ait manqué à la fille d'Henri le Grand ; Anne la magnanime, la pieuse (...) 
la reçut d'une manière convenable à la majesté des deux reines. Mais les affaires du Roi ne permettant pas que cette sage régente pût proportionner le remède au mal, jugez de l'état de ces deux princesses (Henriette et sa fille).

Bossuet (Oraison funèbre d'Henriette de France)

(12) Racine se vante d'avoir, même en une comédie, réjoui le parterre « sans qu'il lui ait coûté une seule de sales équivoques et de ces malhonnêtes plaisanteries qui coûtent maintenant si peu à la plupart de nos écrivains" (...) Ce n'est pas que Racine, dans son œuvre, ait partout et toujours cherché le mot noble, comme le lui reprochent les romantiques (...) Mais, en dépit de ces hardiesses (...) il témoigne à l'égard des mots déshonnêtes (...) une réserve extrême.

F. Brunot (H.L.F., IV, 1, 302-303)

(13) Depuis, le vétéran de la diplomatie mondiale éprouvait quelque peine à redessiner une politique extérieure pour l'Allemagne unie (...) Non que les dogmes fondateurs ne demeurent (...) Mais les trois piliers traditionnels de la diplomatie allemande ont été ébranlés.

Le Monde, 2 Mai 1992

(14) Le quai d'Orsay accuse le coup. Non que le tort causé à l'image de la France à l'étranger soit ressenti comme irréparable : on déplore tout juste quelques sarcasmes des amis britanniques (...) Non que la controverse soit engagée sur l'attitude de la France à l'égard des Palestiniens : l'histoire dira peut-être un jour ce que la venue à Paris du leader du FPLP avait à voir avec une politique qui...

Le Monde, 22 Février 1992

(15) E. Balladur. Assurément le plus européens des dirigeants du RPR. Non pas qu'E.

Balladur puisse être considérée comme un Euromaniaque.

Le Nouvel Observateur, 23-29 Avril 1992.

(16) Non que par moy soit arrogance prinse,

Non que ce soit par curieuse emprinse

D'escrire au Roy ; pour tout cela ma plume

D'ardent désir de voler ne s'allume.

Mon triste deuil seulement l'a contraincte

De faire à vous, et non de vous complaincte.

Marot (Epître au Roy pour succéder en l'estat de son père)

(Vers $1 \grave{a}$ 6)

(17) Chanson « C'est mon homme" :

«C'est pas qu'il est beau,

Qu'il est riche, ni costaud,

Mais je l'aime »

(18) C'est pas que ça m'arrange, mais je peux me débrouiller.

(19) Bon, c'est pas que je m'ennuie, mais il faut que je m'en aille.

(20) Chanson (Y. Montand, années 50) :

"C'est pas qu'elle est belle, belle,

Elle a pas d'bijoux, ni de fines dentelles, 
Mais elle a en elle

Quelque chose qui plaît »

(21) Les leaders des territoires occupés pourront désormais se réclamer ouvertement de la centrale dirigée par M. Yasser Arafat. Ce n'est pas d'ailleurs qu'ils se gênaient, mais enfin, théoriquement, M. Fayçal Husseini et Mme Hanan Achraoui risquaient la prison. Le Monde, 28-29 Juin 1992

(22) L'acceptabilité est à première vue un concept non théorique ; elle n'est rien d'autre qu'une valeur. Cela n'empêche pas le linguiste d'avoir à construire une représentation adéquate du type de jugement correspondant, même s'il le fait de manière discutable (...) Ce n'est pas pour autant que la grammaire devient $\mathrm{T}-1$ normative ; on peut toujours considérer qu'il s'agit là de constatations empiriques.

S. Auroux, "La linguistique est une science normative" Le Langage comme défi, Paris VIII, 1992

(23) - C'est une question de biorythme

- Tu crois au biorythme?

- Ce n'est pas que j'y croie (crois). Cela existe.

(24) - Il est bête?

- Ce n'est pas qu'il soit bête. Il est maladroit.

(25) Quant à la Nomenklatura, ce n'est pas qu'elle n'aime pas Gorbatchev, c'est qu'elle le hait.

(26) S'il trouve des mots aussi justes pour réduire à coups de sourires son cancer à une banalité du destin (...), ce n'est pas parce qu'il puise dans le terreau de son savoir-faire en cherchant une fois de plus à désarmer ceux qui lui reprochent le confort de sa solitude souveraine.

Le Monde, 11-11-1992

(27) Ce n'est pas à dire qu'une langue ne porte pas les caractères correspondant à ceux de la collectivité qui l'a créée, mais...

Séchehaye, "La pensée et la langue".

(28) Ce n'est pas que non négatif ( = «néanmoins, cela n'empêche pas que") :

a) Il leur fait servir à boire par un héraut (...) Ce n'est pas qu'il y admet encore d'autres valets.

Racine (Remarques sur l'Odyssée)

b) Si le titre ne vous plaît, changez-le ; ce n'est pas qu'il m'a paru le plus convenable.

Racine, Lettres

c) Ce n'est pas qu'il faut quelquefois pardonner à celui qui, avec un grand cortège, un habit riche et un magnifique, s'en croit plus de naissance et plus d'esprit : il lit cela dans la contenance et dans les yeux de ceux qui lui parlent.

La Bruyère (Du mérite personnel, 27) 
NB : ont été repris à Brunot : $11,27 \mathrm{a}, \mathrm{b}, \mathrm{c}$, à Grevisse : 5, 6, 10,27b, c, à Guillaume : 4, à

Gaatone : 2, 3, 7, 8, 9, 9, à Gougenheim : 16 .

\section{NOTES}

1. R. Martin me suggère l'interprétation suivante : il ne s'agirait pas du même ce n'est pas que que celui dont je parle ici, mais de ce n'est pas + un que valant sinon. Effectivement on trouve jusqu'au 17e s. un que exceptif après une négation totale ou une interrogation. Dans ce cas, on devrait comprendre quelque chose comme : «Il n'y a rien d'autre à dire sinon que...», « Ne cherchons pas d'autre raison sinon que... ».

2. C'est D. Leeman qui a attiré mon attention sur ce point.

3. Nous laisserons de côté la question de savoir si cet énoncé n'est pas le strict équivalent d'un énoncé affirmatif par sa valeur propre. C'est le problème de la situation de la négation «descriptive » en face de la négation «polémique ». Nous parlons plus loin des rapports entre c'est que ne pas et ce n'est pas que.

\section{RÉSUMÉS}

La forme négative ce n'est pas que, qui est parfois en concurrence avec non que, pose de nombreux problèmes : les principaux sont les rapports entre ce n'est pas que et c'est que, surtout quand ce dernier est suivi d'une négation, et la valeur ou les valeurs de cette tournure. En général, on la décrit comme causale ( Ce n'est pas parce que»), mais un large relevé d'exemples montre que ce n'est pas systématique. Par contre, en regardant les choses de près, on peut lui attribuer toutes les valeurs de ne pas, y compris lorsque ce dernier n'est pas «contre-argumentatif " ou contradictoire («Ce n'est pas qu'il ne l'aime pas, il le déteste»). Mais le plus souvent ce n'est pas que a bien un rôle contre-argumentatif.

The french negative phrase ce n'est pas que leads to many problems, mainly the relationship between this phrase and c'est que, specially when the latter is followed by a negation, and the values of that phrase. Most people describe it as a causal phrase (= « ce n'est pas parce que »), but selecting from a wide range of exemples, one can see that this does not occur on a regular basis. On the other hand, by closely considering the facts, we can attribute every value of ne pas to ce n'est pas que, even the "metalinguistic » one (ce n'est pas qu'il ne l'aime pas, il le déteste). But mostly ce n'est pas que plays a « counter-argumentative » role.

\section{AUTEUR}

\section{PIERRE ATTAL}

Université Paris X 